\title{
On the Generalized LEBESGUE-Ramanujan-Nagell Equation
}

\author{
Ran Yinxia* \\ Longnan Teachers College, Department of Mathematics, Gansu Longnan, China. \\ * Corresponding author. Tel.: 18993930265; email: ranyinxia@163.com \\ Manuscript submitted July 12, 2016; accepted January 5, 2017. \\ doi: 10.17706/ijapm.2017.7.2.112-117
}

\begin{abstract}
Let $p$ is a prime, we studied the the generalized Lebesgue-Ramanujan-Nagell equation. By using the elementary method and algebraic number theory, we obtain one necessary condition which the equation has integer solutions and some sufficient conditions which the equation has no integer solution. 1). Let $x$ be an odd number, one necessary condition which the equation has integer solutions is that $2^{n(p-1)}-1 / p$ contains some square factors. 2). Let $x$ be an even number, when $n=p k(k \geqslant 1)$, all integer solutions for the equation are $(x, y)=\left(0,4^{k}\right)$; when $n=p k+(p-1) / 2(k \geqslant 0)$, all integer solutions are $\left( \pm 2^{p k+(p-1) / 2}, 2^{2 k+1}\right)$; when $n \equiv 1,2,3, \ldots,(p-3) / 2,(p+1) / 2, \ldots, p-1(\bmod p)$, the equation has no integer solution.
\end{abstract}

Key words: Exponential Diophantine equation, integer solutions, integer ring, algebraic number theory.

\section{Introduction}

Let $\mathbf{N}, \mathbf{Z}$ be the set of all positive integers and all integers respectively. In this paper, we deal with the solutions $(x, y)$ of diophantine equation

$$
A x^{2}+B=y^{m}, \quad m \equiv 1(\bmod 2), m>1, x, y, m \in \mathrm{N}
$$

where $A, B$ are positive integers and $A$ is nonsquare. Some special cases of (1) have been settled. When $A=1$, $B=1$ lebsgue [1] has proved that (1) has no integer solution, when $A=2, B=1, n=5$, Nagell [2] has proved that (1) has only integer solutions $(x, y)=( \pm 11,3)$; When $A=1, B=4^{n}, m=7$, and $n=1,2,3,4$ (see [3]-[6]), it has been proved that (1) has no integer solution.

However, when $B=c^{k}$, it is more difficult to solve it. In particular, when $B=p^{k}$, It is a hot research field recently. And, at present these research results were achieved as follow:

1) When $p=2$, Cohn[1,2], Arif and Abu Muriefah[3], Le[4] have gotten all solutions of the equation $x^{2}+2^{m}=y^{n}, \operatorname{gcd}(x, y)=1, n>2:$

- When $m$ is odd, the equation has only two solutions $(x, y, m, n)=(5,3,1,3)$ and $(7,3,5,4)$.

- When $m$ is even, the equation has only one solution $(x, y, m, n)=(11,5,2,3)$.

2) When $p=3, \operatorname{Cohn}[1,2]$, Arif and Abu Muriefah[5,6],luca[7],Tao[8] have gotten all solutions of the 
equation $x^{2}+3^{m}=y^{n},(x, y)=1, n>2$.

3) When $p=5$, Arif and Abu Muriefah [9], [10] and Tao [11] have gotten all solutions of the equation $x^{2}+5^{m}=y^{n},(x, y)=1, n>2$, and $2 \mid m$. Unfortunately, it failed to give the solutions of $2 \mid m$.

4) When $p=7$, Silksek and Cremona [12], Bugeaud, Mignotte and Silksek [13], Luca [14], Huilin-Zhu and Maohua-Le [15] have gotten all solutions of the equation $x^{2}+5^{7}=y^{n}, \quad(x, y)=1, n>2$, and $2 \mid m$. And, when $2 \mid m$, they only got the solutions of $p=11,19,43,67,163$.

Here, we study the solution of $x^{2}+4^{n}=y^{p}$, where $p$ is a prime, and give the following conclusions:

Theorem When $A=1, B=4^{n}, m=p$, the following conclusions will be established:

1) Let $x$ be an odd number, one necessary condition which the equation (1) has integer solutions is that $2^{n(p-1)}-1 / p$ contains some square factors.

2) Let $x$ be an even number, if $n \equiv 0(\bmod p)$, that is $n=p k(k \geq 1)$, all integer solutions for the equation are $(x, y)=\left(0,4^{k}\right)$; if $n \equiv \frac{p-1}{2}(\bmod p)$, that is $n=p k+\frac{p-1}{2}(k \geq 0)$, all integer solutions are $\left( \pm 2^{p k+\frac{p-1}{2}}, 2^{2 k+1}\right)$; if $n \equiv 1,2,3, \cdots, \frac{p-3}{2}, \frac{p+1}{2}, \cdots p-1(\bmod p)$, the equation has no integer solution.

\section{Preliminaries}

Lemma 1 [7] Let $M$ is a unique factorization domain, $k$ is a positive integer, $k \geq 2$, and $\alpha, \beta \in M$, $(\alpha, \beta)=1$, and if $\alpha \beta=\gamma^{k}, \gamma \in M$, then $\alpha=\varepsilon_{1} \mu^{k}, \beta=\varepsilon_{2} v^{k}, \mu, v \in M$, and $\varepsilon_{1} \varepsilon_{2}=\varepsilon^{k}$, where $\varepsilon_{1}, \varepsilon_{2}, \varepsilon$ are units in $M$.

Lemma 2 For the diophantine equation $x^{2}+1=2^{k} y^{p}$, there are following conclusions:

1) If $k=0$, then the equation only has integer solution $(x, y)=(0,1)$;

2) If $k=1$, then the equation only has integer solutions $(x, y)=( \pm 1,1)$;

3) If $k=2,3, \cdots, p-1$, then all equations have no integer solutions.

proof: 1), 2) By lemma 1, it is easy to prove;

Obviously, $x$ is an odd number, then $x^{2} \equiv 1(\bmod 4)$ and $x^{2}+1 \equiv 2(\bmod 4)$, But if $k=2,3, \cdots, p-1$, then $x^{2}+1=2^{k} y^{p} \equiv 0(\bmod 4)$, This is a contradiction. So $x^{2}+1=2^{k} y^{p}, \quad(k=2,3, \cdots, p-1)$ has no integer solutions.

Lemma 3 When $p \equiv 1(\bmod 4)$, if $k \equiv 0,1(\bmod 4)$, then $C_{p}^{k}(k \geq 0, k \in Z)$ is odd numbers, and if $k \equiv 2,3(\bmod 4)$, then $C_{p}^{k}(k \geq 0, k \in Z)$ is even numbers; when $p \equiv 3(\bmod 4)$, if $k \equiv 1,3(\bmod 8)$, then $C_{p}^{k}(k \geq 0, k \in \mathrm{Z})$ is odd numbers, and if $k \equiv 5,7(\bmod 8)$, then $C_{p}^{k}(k \geq 0, k \in \mathrm{Z})$ is even numbers.

Lemma 4 If $p$ is a prime, and $(a, p)=1$, then $a^{p-1} \equiv 1(\bmod p)$.

\section{Proof of Theorem}


1) First, suppose $x \equiv 1(\bmod 2)$, in $Z[i], x^{2}+4^{n}=y^{p}$ can be decomposed into as follows

$$
\left(x+2^{n} i\right)\left(x-2^{n} i\right)=y^{p}, x, y \in Z \text {. }
$$

Let $\delta=\left(x+2^{n} i, x-2^{n} i\right)$, because of $\delta \mid\left(2 x, 2^{n+1} i\right)=2, \quad \delta$ can only be $1,1+i, 2$. But $x \equiv 1(\bmod 2), \quad$ so $\quad x+2^{n} \equiv 1(\bmod 2) \quad$, then $\quad \delta \neq 2 \quad$. If $\delta=1+i \quad$, then $2=N(1+i) \mid N\left(x+2^{n} i\right)=x^{2}+2^{2 n}$. However $x \equiv 1(\bmod 2)$, So the integer $x$ does not exist. As a result, $\delta=1$. Thus ,by lemma 1, $x+2^{n} i=(a+b i)^{p}, x, a, b \in \mathrm{Z}$,

If $p \equiv 1(\bmod 4)$, then

$$
\begin{aligned}
& x=a^{p}-C_{p}^{2} a^{p-2} b^{2}+C_{p}^{4} a^{p-4} b^{4}-C_{p}^{6} a^{p-6} b^{6}+\cdots-C_{p}^{p-7} a^{7} b^{p-7}+C_{p}^{p-5} a^{5} b^{p-5}-C_{p}^{p-3} a^{3} b^{p-3}+C_{p}^{p-1} a b^{p-1} \\
& 2^{n}=b\left(C_{p}^{1} a^{p-1}-C_{p}^{3} a^{p-3} b^{2}+C_{p}^{5} a^{p-5} b^{4}-C_{p}^{7} a^{p-7} b^{6}+\cdots+C_{p}^{p-4} a^{4} b^{p-5}-C_{p}^{p-2} a^{2} b^{p-3}+b^{p-1}\right) .
\end{aligned}
$$

If $p \equiv 3(\bmod 4)$, then

$$
\begin{aligned}
& x=a^{p}-C_{p}^{2} a^{p-2} b^{2}+C_{p}^{4} a^{p-4} b^{4}-C_{p}^{6} a^{p-6} b^{6}+\cdots+C_{p}^{p-7} a^{7} b^{p-7}-C_{p}^{p-5} a^{5} b^{p-5}+C_{p}^{p-3} a^{3} b^{p-3}-C_{p}^{p-1} a b^{p-1} \\
& 2^{n}=b\left(C_{p}^{1} a^{p-1}-C_{p}^{3} a^{p-3} b^{2}+C_{p}^{5} a^{p-5} b^{4}-C_{p}^{7} a^{p-7} b^{6}+\cdots-C_{p}^{p-4} a^{4} b^{p-5}+C_{p}^{p-2} a^{2} b^{p-3}-b^{p-1}\right) .
\end{aligned}
$$

So $b= \pm 1, \pm 2^{t}(1 \leq t \leq n-1), \pm 2^{n}$.

If $b= \pm 1$, When $p \equiv 1(\bmod 4)$,

then $C_{p}^{1} a^{p-1}-C_{p}^{3} a^{p-3}+C_{p}^{5} a^{p-5}-C_{p}^{7} a^{p-7}+\cdots+C_{p}^{p-4} a^{4}-C_{p}^{p-2} a^{2}= \pm 2^{n}-1$, so $a$ must be odd.

Let $p=4 k+1$, by lemma3, $C_{p}^{1}, C_{p}^{5}, C_{p}^{9}, \cdots C_{p}^{p-8}, C_{p}^{p-4}$, these $\mathrm{k}$ integer numbers are odd, and $C_{p}^{3}, C_{p}^{7}, C_{p}^{11}, \cdots C_{p}^{p-6}, C_{p}^{p-2}$, these $\mathrm{k}$ integer numbers are even. Thus, if $k$ is even, the equation $C_{p}^{1} a^{p-1}-C_{p}^{3} a^{p-3}+C_{p}^{5} a^{p-5}-C_{p}^{7} a^{p-7}+\cdots+C_{p}^{p-4} a^{4}-C_{p}^{p-2} a^{2}= \pm 2^{n}-1$ doesn't set up; and if $k$ is odd, $x=a^{p}-C_{p}^{2} a^{p-2} b^{2}+C_{p}^{4} a^{p-4} b^{4}-C_{p}^{6} a^{p-6} b^{6}+\cdots-C_{p}^{p-7} a^{7} b^{p-7}+C_{p}^{p-5} a^{5} b^{p-5}-C_{p}^{p-3} a^{3} b^{p-3}+C_{p}^{p-1} a b^{p-1} \quad$ is even, this contradict with $x \equiv 1(\bmod 2)$, in fact, $C_{p}^{0}, C_{p}^{4}, C_{p}^{8}, \cdots C_{p}^{p-5}, C_{p}^{p-1}$, these $\mathrm{k}+1$ integer numbers are odd, $C_{p}^{2}, C_{p}^{6}, C_{p}^{10}, \cdots C_{p}^{p-7}, C_{p}^{p-3}$, these k integer numbers are even, so $x$ is even.

When $p \equiv 3(\bmod 4)$, then $C_{p}^{1} a^{p-1}-C_{p}^{3} a^{p-3}+C_{p}^{5} a^{p-5}-C_{p}^{7} a^{p-7}+\cdots-C_{p}^{p-4} a^{4}+C_{p}^{p-2} a^{2}= \pm 2^{n}-1$, so $a$ must be odd.

Let $p=8 k+3$, by lemma3, $C_{p}^{1}, C_{p}^{3}, C_{p}^{9}, C_{p}^{11}, C_{p}^{17}, C_{p}^{19}, \cdots, C_{p}^{p-12}, C_{p}^{p-10}, C_{p}^{p-4}, C_{p}^{p-2}$ are odd integer numbers, and $C_{p}^{5}, C_{p}^{7}, C_{p}^{13}, C_{p}^{15} \cdots C_{p}^{p-8}, C_{p}^{p-6}$ are even integer numbers. Thus,

$C_{p}^{1} a^{p-1}-C_{p}^{3} a^{p-3}+C_{p}^{5} a^{p-5}-C_{p}^{7} a^{p-7}+\cdots-C_{p}^{p-4} a^{4}+C_{p}^{p-2} a^{2}$ is even, however, $\pm 2^{n}-1$ is odd. 
Let $p=8 k+7$, by lemma3, $C_{p}^{1}, C_{p}^{3}, C_{p}^{9}, C_{p}^{11}, C_{p}^{17}, C_{p}^{19}, \cdots, C_{p}^{p-12}, C_{p}^{p-10}, C_{p}^{p-4}, C_{p}^{p-2}$ are odd integer numbers, and $C_{p}^{5}, C_{p}^{7}, C_{p}^{13}, C_{p}^{15} \cdots, C_{p}^{p-9}, C_{p}^{p-8}, C_{p}^{p-2}$ are even integer numbers. Thus,

$C_{p}^{1} a^{p-1}-C_{p}^{3} a^{p-3}+C_{p}^{5} a^{p-5}-C_{p}^{7} a^{p-7}+\cdots-C_{p}^{p-4} a^{4}+C_{p}^{p-2} a^{2}$ is even, however, $\pm 2^{n}-1$ is odd.

If

$$
b= \pm 2^{t}(1 \leq t \leq n-1)
$$

then

$C_{p}^{1} a^{p-1}-C_{p}^{3} a^{p-3} b^{2}+C_{p}^{5} a^{p-5} b^{4}-C_{p}^{7} a^{p-7} b^{6}+\cdots \pm C_{p}^{p-4} a^{4} b^{p-5} \mp C_{p}^{p-2} a^{2} b^{p-3} \pm b^{p-1}= \pm 2^{n-t}$, so $a$ is even.

Thus

$x=a^{p}-C_{p}^{2} a^{p-2} b^{2}+C_{p}^{4} a^{p-4} b^{4}-C_{p}^{6} a^{p-6} b^{6}+\cdots \mp C_{p}^{p-7} a^{7} b^{p-7} \mp C_{p}^{p-5} a^{5} b^{p-5} \mp C_{p}^{p-3} a^{3} b^{p-3} \pm C_{p}^{p-1} a b^{p-1}$

is even, this contradict with $x \equiv 1(\bmod 2)$;

If $b=-2^{n}$, When $p \equiv 1(\bmod 4)$,

$C_{p}^{1} a^{p-1}-C_{p}^{3} a^{p-3} b^{2}+C_{p}^{5} a^{p-5} b^{4}-C_{p}^{7} a^{p-7} b^{6}+\cdots+C_{p}^{p-4} a^{4} b^{p-5}-C_{p}^{p-2} a^{2} b^{p-3}+b^{p-1}=-1 \quad$ that $\quad$ is

$C_{p}^{1} a^{p-1}-C_{p}^{3} a^{p-3} b^{2}+C_{p}^{5} a^{p-5} b^{4}-C_{p}^{7} a^{p-7} b^{6}+\cdots+C_{p}^{p-4} a^{4} b^{p-5}-C_{p}^{p-2} a^{2} b^{p-3}=-1-2^{(p-1) n} \quad$,

$2^{(p-1) n} \equiv-1(\bmod p)$, but, indeed, by lemma $4,2^{(p-1) n} \equiv 1(\bmod p)$;

When $p \equiv 3(\bmod 4)$,

$$
C_{p}^{1} a^{p-1}-C_{p}^{3} a^{p-3} b^{2}+C_{p}^{5} a^{p-5} b^{4}-C_{p}^{7} a^{p-7} b^{6}+\cdots-C_{p}^{p-4} a^{4} b^{p-5}+C_{p}^{p-2} a^{2} b^{p-3}-b^{p-1}=-1,
$$

that is $C_{p}^{1} a^{p-1}-C_{p}^{3} a^{p-3} b^{2}+C_{p}^{5} a^{p-5} b^{4}-C_{p}^{7} a^{p-7} b^{6}+\cdots-C_{p}^{p-4} a^{4} b^{p-5}+C_{p}^{p-2} a^{2} b^{p-3}=2^{(p-1) n}-1$,

so $a^{2}\left(a^{p-3}-\frac{C_{p}^{3}}{p} a^{p-5} b^{2}+\frac{C_{p}^{5}}{p} a^{p-7} b^{4}-\frac{C_{p}^{7}}{p} a^{p-9} b^{6}+\cdots-\frac{C_{p}^{p-4}}{p} a^{2} b^{p-5}+\frac{C_{p}^{p-2}}{p} a b^{p-3}\right)=2^{(p-1) n-1} / p^{\prime}$

thus only when $2^{(p-1) n-1} / p$ contains some square factors, the equation may have integer solutions.

If $b=2^{n}$, When $p \equiv 1(\bmod 4)$,

$$
C_{p}^{1} a^{p-1}-C_{p}^{3} a^{p-3} b^{2}+C_{p}^{5} a^{p-5} b^{4}-C_{p}^{7} a^{p-7} b^{6}+\cdots+C_{p}^{p-4} a^{4} b^{p-5}-C_{p}^{p-2} a^{2} b^{p-3}+b^{p-1}=1,
$$

that is $C_{p}^{1} a^{p-1}-C_{p}^{3} a^{p-3} b^{2}+C_{p}^{5} a^{p-5} b^{4}-C_{p}^{7} a^{p-7} b^{6}+\cdots+C_{p}^{p-4} a^{4} b^{p-5}-C_{p}^{p-2} a^{2} b^{p-3}=1-2^{(p-1) n}$, so $-a^{2}\left(a^{p-3}-\frac{C_{p}^{3}}{p} a^{p-5} b^{2}+\frac{C_{p}^{5}}{p} a^{p-7} b^{4}-\frac{C_{p}^{7}}{p} a^{p-9} b^{6}+\cdots+\frac{C_{p}^{p-4}}{p} a^{2} b^{p-5}-\frac{C_{p}^{p-2}}{p} a b^{p-3}\right)=2^{(p-1) n-1} / p^{\prime}$, thus only when $2^{(p-1) n-1} / p$ contains some square factors, the equation may have integer solutions.

When $p \equiv 3(\bmod 4)$,

$$
C_{p}^{1} a^{p-1}-C_{p}^{3} a^{p-3} b^{2}+C_{p}^{5} a^{p-5} b^{4}-C_{p}^{7} a^{p-7} b^{6}+\cdots-C_{p}^{p-4} a^{4} b^{p-5}+C_{p}^{p-2} a^{2} b^{p-3}-b^{p-1}=1,
$$


that is $C_{p}^{1} a^{p-1}-C_{p}^{3} a^{p-3} b^{2}+C_{p}^{5} a^{p-5} b^{4}-C_{p}^{7} a^{p-7} b^{6}+\cdots-C_{p}^{p-4} a^{4} b^{p-5}+C_{p}^{p-2} a^{2} b^{p-3}=2^{(p-1) n}+1$, so $2^{(p-1) n} \equiv-1(\bmod p)$, but, indeed, by lemma $4,2^{(p-1) n} \equiv 1(\bmod p)$;

So, when $x \equiv 1(\bmod 2)$, one necessary condition which the equation has integer solutions is that $2^{(p-1) n-1} / p$ contains some square factors.

2) Second, suppose $x \equiv 0(\bmod 2)$, thus $y \equiv 0(\bmod 2)$. Now make $x=2 x_{1}, y=2 y_{1}$, then the equation can be turned into $x_{1}^{2}+4^{n-1}=2^{p-2} y_{1}^{p}$, obviously $x_{1} \equiv 0(\bmod 2)$, then make $x_{1}=2 x_{2}$, it can be $x_{2}{ }^{2}+4^{n-2}=2^{p-4} y_{1}{ }^{p}$, also make $x_{2}=2 x_{3}$ again, it can be $x_{3}{ }^{2}+4^{n-3}=2^{p-6} y_{1}{ }^{p}, \ldots$, make $x_{\frac{p-3}{2}}=2 x_{\frac{p-1}{2}}$ again, it can be $x_{\frac{p-1}{2}}{ }^{2}+4^{n-\frac{p-1}{2}}=2 y_{1}^{p}$, now make $x_{\frac{p-1}{2}}=2 x_{\frac{p+1}{2}}, y_{1}=2 y_{2}$ it can be $x_{\frac{p+1}{2}}{ }^{2}+4^{n-\frac{p+1}{2}}=2^{p-1} y_{2}^{p}$, then make $x_{\frac{p+1}{2}}=2 x_{\frac{p+3}{2}}$ again, it can be $x_{\frac{p+3}{2}}{ }^{2}+4^{n-\frac{p+3}{2}}=2^{p-3} y_{2}{ }^{p}, \ldots$, make $x_{p-1}=2 x_{p}$ again, it can be $x_{p}^{2}+4^{n-p}=y_{2}{ }^{p}$, where $x_{1}, x_{2}, \cdots, x_{p}, y_{1}, y_{2} \in Z$.

According to such substituted method, it can be concluded:

When $n \equiv 1(\bmod p)$, the original equation is equivalent to solving $x^{2}+4=y^{p}$, and according to the above-mentioned regularity, it is finally equivalent to solving $x^{2}+1=2^{p-2} y^{p}$; When $n \equiv 2(\bmod p)$, it is equivalent to solving $x^{2}+4^{2}=y^{p}$, and according to the same regularity, it is finally equivalent to solving $x^{2}+1=2^{p-4} y^{p} ;$ When $n \equiv 3(\bmod p)$, it is equivalent to solving $x^{2}+4^{3}=y^{p}$, and according to the same regularity, it is finally equivalent to solving $x^{2}+1=2^{p-6} y^{p} ; \ldots$, When $n \equiv \frac{p-1}{2}(\bmod p)$, it is equivalent to solving $x^{2}+4^{\frac{p-1}{2}}=y^{p}$, and according to the same regularity, it is finally equivalent to solving $x^{2}+1=2 y^{p}$; When $n \equiv \frac{p+1}{2}(\bmod p)$, it is equivalent to solving $x^{2}+4^{\frac{p+1}{2}}=y^{p}$, and according to the same regularity, it is equivalent to solving $x^{2}+1=2^{p-1} y^{p} ; \ldots$, When $n \equiv p-1(\bmod p)$, it is equivalent to solving $x^{2}+4^{p-1}=y^{p}$, and according to the same regularity, it is finally equivalent to solving $x^{2}+1=2^{2} y^{p}$; When $n \equiv 0(\bmod p)$, the original equation is equivalent to solving $x^{2}+4^{p}=y^{p}$, and according to the same regularity, it is finally equivalent to solving $x^{2}+1=y^{p}$. Therefore, by lemma2, when $n \equiv 1,2,3, \cdots, \frac{p-3}{2}, \frac{p-1}{2}, \cdots, p-1(\bmod p)$, the equation has no integer solutions;

When $n \equiv 0, \frac{p-1}{2}(\bmod p)$, the equation has integer solutions, and when $n \equiv 0(\bmod p)$ that is $n=p k(k \geq 1)$, solutions of the equation will must be $(x, y)=\left(0,4^{k}\right) ;$ if $n \equiv \frac{p-1}{2}(\bmod p)$, that is 
$n=p k+\frac{p-1}{2}(k \geq 0)$, all integer solutions are $\left( \pm 2^{p k+\frac{p-1}{2}}, 2^{2 k+1}\right)$.

\section{References}

[1] Lebsgue, V. A. (1850). Surlimpossibiliteen numbers entiers de equation $x^{m}=y^{2}+1$. Nouv. Amn. Math.

[2] Nagell, T. (1921). Sur limpossibilite de quelques equations deux indeterminess. Norsk Marem Forenings Skrifter Sene l.

[3] Na, L. (2011). Science Technology and Engineering.

[4] Li, G., \& Ma, Y. G. (2008). Journal of Southwest University for Nationalities.

[5] Ran, Y. X. (2012). Journal of southwest university for nationalities, 38.

[6] Ran, Y. X. (2012). Journal of Yan An University, 31.

[7] Pan, C. D., \& Pan, C. P. (2003). Algebraic Number Theory. Shandong: Shandong University Press.

[8] Cohn, J. H. E. (1992). The Diophantine equation $x^{2}+2^{k}=y^{n}$. Arch. Math. (Basel), 59(4), 341-344.

[9] Cohn, J. H. E. (1999). The Diophantine equation $x^{2}+2^{k}=y^{n}$, II. Int. J. Math. Math. Sci., 22(3), 459-462.

[10] Arif, S. A., Muriefah, F. S. (1997). On the Diophantine equation $x^{2}+2^{k}=y^{n}$. Int. J. Math. Math. Sci., 20(2), 299-304.

[11] Le, M. (2002). On Cohn's conjecture concerning the Diophantine equation $x^{2}+2^{m}=y^{n}$. Arch. Math. (Basel), 78(1), 26-35.

[12] Arif, S. A., \& Muriefah, F. S. A. (2002). On the Diophantine equation $x^{2}+q^{2 k+1}=y^{n}$. J. Number Theory, 9595-100.

[13] Bennett, M. A., \& Skinner, C. M. (2004). Ternary Diophantine equation via Galois representations and modular forms. Canad. J. Math., 56, 23-54.

[14] Berczes, A., \& Pink, I. (2008). On the Diophantine equation $x^{2}+p^{2 k}=y^{n}$. Arch. Math., 91, 505-517.

[15] Le, M., \& Hu, Y. (2011). New advancea on the generalized Lebesgue-Ramanujan-Nagell equation[J]. Advances in Mathematics, 41(4), 385-396.

[16] Bennett, M. A., Ellenberg, J. S., \& Ng, N. C. (2010). The Diophantine equation $A^{4}+2 \delta B^{2}=C^{n}$. Int. J. Number Theory, 6(2), 311-338.

[17] Zhu, H. L. (2011). A note on the diophantine equation $x^{2}+q^{m}=y^{3}$. Acta Arith., 146(2), 195-202.

[18] Zhu, H. L., \& Le, M. (2011). On some generalized Ramanujan-Nagell equations. J. Number Theory, 131(3), 458-469.

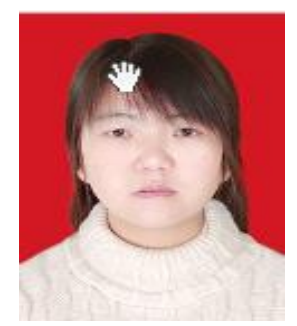

Ran Yinxia was born in April 1983. She comes from Gansu province, China. She graduated from Northwestern University with a master of science degree in 2009, and studied on number theory research. After graduation, she has been taught in Longnan Teachers College. 